\title{
Photo-thermo-acoustic analysis of heterogeneous semiconductor structures under pulse laser irradiation
}

\author{
R. Burbelo ${ }^{1}$, M. Isaiev², A. Kuzmich ${ }^{3}$ \\ Taras Shevchenko Kyiv National University, Physics Department, \\ 64, Volodymyrs'ka str., 01601 Kyiv, Ukraine \\ E-mail:rmb@univ.kiev.ual, isaev@univ.kiev.ua ${ }^{2}, k u z m i c h @ u n i v . k i e v . u a^{3}$
}

\begin{abstract}
The analysis of photo-thermo-acoustic transformation in materials with the modified properties of a surface layer has been made in this work. Formation of a photoacoustic response in a layered structure of the type "implanted layer + crystalline $\mathrm{Si}$ substrate" as a result of its irradiation by one laser pulse with duration of $20 \mathrm{~ns}$ is analyzed.
\end{abstract}

Keywords: photo-acoustics, heterogeneous structure.

Manuscript received 01.10.10; accepted for publication 16.03.11; published online 30.06.11.

\section{Introduction}

The basis of modern materials science is materials inhomogeneous in their structure, in which the region of modification is characterized by the size of nano- and submicron scales. These issues include the structure that in accord with technological needs has significant changes in properties of a subsurface layer, such as epitaxial and porous layers, ranges of doping in semiconductor structures and others.

Clearly, in practice the study of structures with regions of heterogeneity of such magnitude by photoacoustic (PA) methods using the sources of radiation with the intensity modulated by the harmonic law is quite complicated task. For example, during irradiation of silicon with the frequency close to $30 \mathrm{MHz}$ the length of thermal diffusion (the main parameter of resolution in the PA measurements) possesses the value approximately $1 \mu \mathrm{m}$.

On the other hand, it is possible in principle to investigate structures with characteristic sizes at the submicron level, using for excitation of the PA signal by short (about $10 \mathrm{~ns}$ ) laser pulses.

This work presents a mathematical model of photothermo-acoustic transformation in heterogeneous media. Formation of an acoustic signal in a layered structure of the type "implanted layer + crystalline Si substrate" as a result of its irradiation by the laser pulse with duration $20 \mathrm{~ns}$ is analyzed on the base of the model proposed. The case is described when layers of such structure differ only by thermal parameters. The influence of value ratio between the thermal parameters of structure layers on formation of PA response is analyzed. It is shown that in the case of strong absorption, the value of these ratios influences significantly the PA signal even for submicron size structures.

\section{Evolution of temperature distribution in inhomogeneous solids}

Let's consider the following non-stationary heat equation

$\frac{\partial T}{\partial t}=\frac{\partial}{\partial z}\left(D(z) \frac{\partial T}{\partial z}\right)+\frac{I(1-R) \cdot \alpha}{c \rho} \cdot g(t) \cdot e^{-\alpha z}$

where $g(t)$ describes the temporal distribution of the incident light intensity. In the case of a single pulse, $g(t)=H(t)-H(t-\tau), H(t)$ is the Heaviside function.

In all these calculations, we will take $I=$ $1 \mathrm{MW} / \mathrm{cm}^{2}, R=0.37, \tau=20 \mathrm{~ns}, \alpha=5 \cdot 10^{4} \mathrm{~cm}^{-1}, c=$ $0.8 \mathrm{~J} /(\mathrm{g} \cdot \mathrm{K}), \rho=2.3 \mathrm{~g} / \mathrm{cm}^{3}$ as constants and will examine only changes in the thermal diffusivity.

The following boundary conditions are more often realized in practice:

- $\left.(\partial T / \partial z)\right|_{z=0}=0-$ absence of heat outflow from the sample's top surface into external environment;

- $T_{z=z_{\max }}=0-$ contact of the sample's bottom surface with the thermostat $\left(z_{\max }=300 \mu \mathrm{m}-\right.$ thickness of the sample); 
The initial conditions are as follows:

- $\left.T\right|_{t=0}=0$ - the uniform distribution of the temperature in the sample before irradiation (we will accept that the initial temperature is zero without any loss of generality: we will consider only growth over the initial temperature).

For definiteness, the case when the thickness of layer $l$ lies within the limits of 0.5 to $1 \mu \mathrm{m}$ (thickness of the sample $z_{\max }=300 \mu \mathrm{m}$ ) is considered. The value of thermal diffusivity for the top layer is $D_{1}=0.09 \mathrm{~cm}^{2} / \mathrm{s}$ and for crystal-substrate material is $D_{2}=0.94 \mathrm{~cm}^{2} / \mathrm{s}$. The value corresponds to a real situation [1], for example, when surface of the monocrystalline $\mathrm{Si}$ plate is processed (i.e., under modification). The time dependence of surface temperature $(T(0, t))$ for different thicknesses of the modified layer in this structure is shown in Fig. 1. The solid line illustrates $T(0, t)$ for homogeneous samples with $D_{1}$ and $D_{2}$. As one can see, with increasing the layer thickness, surface temperature increases, too (temperature at the heated end for a homogeneous sample and that for the sample with a modified surface layer are different in their magnitude up to $80 \mathrm{~K}$ ), and, as shown in [2,3] (where more detail evolution of temperature distribution is considered), the region of thermal energy localization is reduced.

As seen from this figure, at the initial stage of heating (when $t<l^{2} / D_{1} \quad-$ thermal energy is concentrated in the first layer) the time dependence of surface temperature $(T(0, t))$ has the character similar to that of the curve $(T(0, t))$ for a homogeneous sample with the coefficient of thermal diffusivity $D_{1}$. As heat diffuses into the second layer, the curve of time dependence of surface temperature gradually gets a shape of the curve $T(0, t)$ for a homogeneous sample with the coefficient of thermal diffusivity $D_{2}$, and in the case $t>l^{2} / D_{1}$ they have the same asymptote.

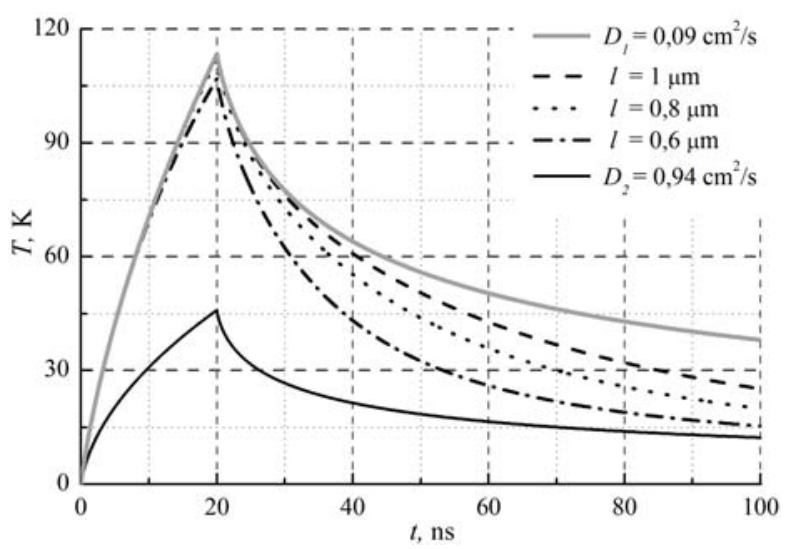

Fig. 1. Time dependence of the surface temperature for twolayer structure with different values of the thickness of modified layer.

\section{Thermo-elastic displacements}

To calculate the elastic displacements, we use the onedimensional thermo-elasticity equation [4]

$$
\frac{\partial^{2} u}{\partial t^{2}}-v^{2} \frac{\partial^{2} u}{\partial z^{2}}=\beta \frac{\partial T}{\partial z},
$$

here $u$ is the elastic displacement inside material (caused by non-equilibrium temperature distribution); $v$ - sound velocity; $\beta=\alpha_{T}(3 \lambda+2 \mu) / \rho ; \lambda$ and $\mu-$ Lame parameters; $\alpha_{T}-$ coefficient of the lattice thermal expansion.

To solve the equation, let us use the following boundary conditions - the bottom surface of the sample is rigidly fixed and the top is free:

$\left\{\begin{array}{l}\left.\frac{\partial u}{\partial z}\right|_{z=0}=0 \\ \left.u\right|_{z=z_{\max }}=0 .\end{array}\right.$

In accordance with the given boundary conditions, the function $u(z, t)$ can be written as follows:

$$
u(z, t)=\sum_{m=0}^{\infty} f_{m}(t) \cos \left(a_{m} z\right), \quad a_{m}=\left(\frac{\pi}{2}+\pi m\right) \frac{1}{z_{\max }} .
$$

Putting (3) into the formula (2), taking into account the boundary conditions to the heat equation (1) and the linear independence of the basic functions $\cos \left(a_{m} z\right)$, we can get the equation for the coefficients $f_{m}(t)$ :

$$
\frac{\mathrm{d}^{2} f_{m}(t)}{\mathrm{d} t^{2}}+v^{2} a_{m}^{2} f_{m}(t)=-\beta \sum_{n=0}^{\infty} a_{n} b_{n}(t) c_{n m},
$$

where

$$
\begin{aligned}
& b_{n}(t)=\frac{2}{z_{\max }} \int_{0}^{z_{\max }} T(z, t) \cdot \cos \left(a_{n} z\right) \mathrm{d} z, \\
& c_{n m}=\frac{2}{z_{\max }} \int_{0}^{z_{\max }} \sin \left(a_{n} z\right) \cdot \cos \left(a_{m} z\right) \mathrm{d} z= \\
& =\left\{\begin{array}{l}
\frac{2}{(m+n+1) \pi}, m+n=2 p, p \in \mathbf{Z} \\
\frac{2}{(n-m) \pi}, m+n=2 p+1, p \in \mathbf{Z} .
\end{array}\right. \\
& \left(\begin{array}{l}
\text { Considering } \quad \text { zero } \quad \text { initial and } \\
\partial u(z, 0)=0 \Rightarrow f_{m}(0)=0
\end{array}\right. \\
& \left.\partial u(z, 0) / \partial t=0 \Rightarrow f_{m}^{\prime}(0)=0\right), \text { we can obtain a solution }
\end{aligned}
$$
of the equation (3) in the form

$$
f_{m}(t)=\frac{\beta}{v} \sum_{n=0}^{\infty} c_{n m} \frac{a_{n}}{a_{m}} \int_{0}^{t} \sin \left(v a_{m}(s-t)\right) \cdot b_{n}(s) \mathrm{d} s,
$$

and, accordingly, for the elastic displacements

$$
u=\frac{\beta}{v} \sum_{m=0}^{\infty} \sum_{n=0}^{\infty} c_{n m} \frac{a_{n}}{a_{m}} \int_{0}^{t} \sin \left(v a_{m}(s-t)\right) \cdot b_{n}(s) \mathrm{d} s \times \cos \left(a_{m} z\right) .
$$




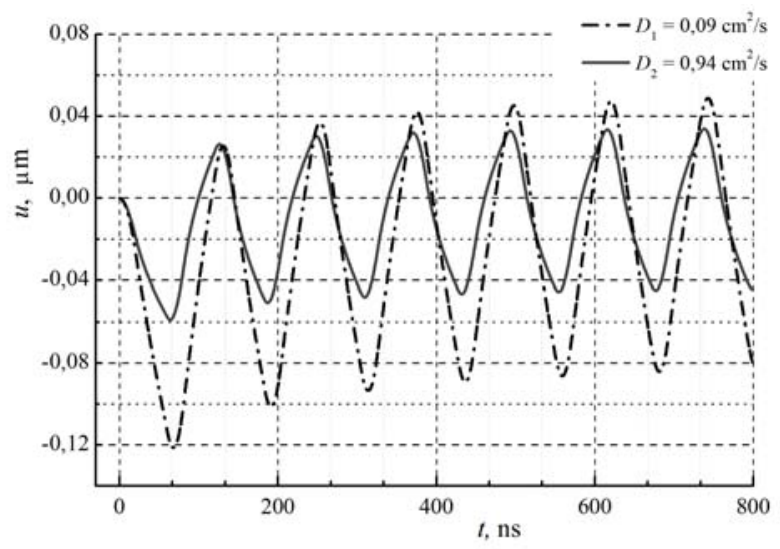

Fig. 2. Elastic displacements of top surface for the samples with different values of the thermal diffusivity coefficient.

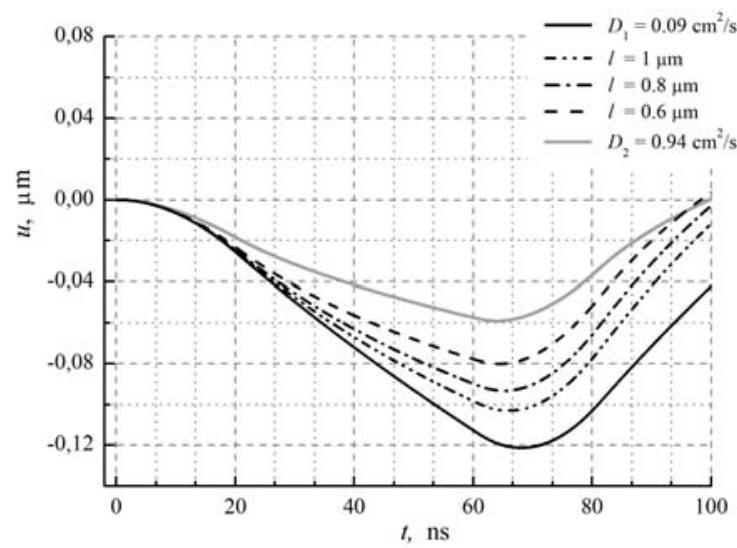

Fig. 3. Time dependence of elastic displacements for the top surface of structures with different thicknesses of the modified layer.

In Fig. 2, the time dependence of surface displacements $(u(0, t))$ for homogeneous samples with different values of the thermal diffusivity coefficients are presented. Fig. 2 shows that the curve $u(0, t)$ has oscillating character with frequencies corresponding to resonance frequencies of the sample, i.e. the oscillating nature of the curves in Fig. 2 is a consequence with multiple reflection of sound waves in the system "top bottom boundary". Fig. 2 shows that oscillation of the function $u(0, t)$ is non-stationary (amplitude and phase shift depend on time), but reaches the stationary mode with time.
In Fig. 3, the time dependence for surface displacements in the structure with the modified subsurface layer is presented. Thicknesses of the layer correspond to the situation that we considered in the item 2. Fig. 3 shows that, with increasing the thickness of the modified layer, the amplitude of displacements increases, which correlates with the time dependence of surface temperature (Fig. 1). As one can see from Fig. 3, after the end of laser pulse, material continues to expand, which is caused by further redistribution of the sources of thermo-elastic stresses (heat diffusion) and with the mass inertia of the system.

\section{Conclusions}

In this work, the mathematical model of photo-thermoacoustic transformation in heterogeneous media is presented. Being based on the proposed model, formation of the acoustic signal in a layered structure of the type "implanted layer + crystalline Si substrate" as a result of its irradiation by the laser pulse with duration $20 \mathrm{~ns}$ is analyzed. The case when layers of this structure differ only by thermal parameters has been described. The influence of value ratio between the thermal parameters of structure layers on formation of the photoacoustic response has been analyzed. It has been shown that in the case of strong absorption, the value of these ratios significantly influences the photo-acoustic signal even for submicron size structures.

\section{References}

1. A. Okhotin, Thermal Conduction of Solids (Handbook). Energoatomizdat, Moscow, 1984 (in Russian).

2. R. Burbelo, M. Isaiev, A. Kuzmich, Evolution of temperature distribution in implanted Si-based structures: pulse mode of laser irradiation // Ukr. J. Phys., 55 (3), p. 317-321 (2010).

3. R. Burbelo, M. Isaiev, A. Kuzmich, Photothermal analysis of heterogeneous semiconductor structures under pulse laser irradiation // Semiconductor Physics, Quantum Electronics \& Optoelectronics, 12(4), p. 403-405 (2009).

4. A. Kovalenko, Thermoelasticity. Vyshcha Shkola, Kyiv, 1975 (in Russian). 